\title{
COMPOSTOS ISOLADOS DE JATROPHA ISABELLI (MÜELL ARG.) COM ATIVIDADE GASTROPROTETORA
}

\author{
Janaina Kieling Fröhlich*, Aline Augusti Boligon*, Andrieli Cassel \\ Feltrin*, Vanessa Janovik*, Amanda Luana Forbrig Froeder**, \\ Margareth Linde Athayde*** \\ Universidade Federal de Santa Maria
}

RESUMO: As plantas medicinais são importantes para a pesquisa farmacológica e para o desenvolvimento de fármacos, não somente quando seus constituintes são usados diretamente como agentes terapêuticos, mas também como matérias-primas isoladas para a síntese ou modelo de compostos farmacologicamente ativos. No entanto, apenas uma pequena parte das plantas foi estudada e tiveram suas ações farmacológicas conhecidas. A fim de mostrar o potencial de Jatropha isabelli Müell Arg (Euphorbiaceae) para o desenvolvimento de um medicamento a partir de seus ativos isolados, as atividades gastroprotetoras da planta são apresentadas.

Descritores: Jatropha isabelli; Euphorbiaceae; Yagua rova; Atividade gastroprotetora.

\section{COMPOUNDS ISOLATED FROM JATROPHA ISABELLI (MÜELL ARG.) WITH} GASTROPROTECTIVE ACTIVITY

\begin{abstract}
The medicinal plants are important for the pharmacological research and for the development of drugs, not only when their constituents are used directly as terapeutic agents but also as isolated raw materials for the synthesis or as model of active pharmacologically compounds. However, only a small part of these plants was studied and its pharmacological actions were known. In order to show the potential of Jatropha isabelli Müell Arg. (Euphorbiaceae) to development of a medicine from its isolated actives, the gastroprotective activities of the plant are presented.
\end{abstract}

Descriptors: Jatropha isabelli; Euphorbiaceae; Yagua rova; Gastroprotective activity.

\footnotetext{
* Mestranda do Programa de Pós-Graduação em Ciências Farmacêuticas da Universidade Federal de Santa Maria, Santa Maria, RS.

** Aluna da Graduação do Curso de Farmácia da Universidade Federal de Santa Maria.

*** Professora Adjunta do Departamento de Farmácia Industrial da Universidade Federal de Santa Maria, Santa Maria, RS. ISSN 2236-5843
} 


\section{Introdução}

A biodiversidade dos vegetais constitui uma grande riqueza em potencial para a saúde humana, sendo as plantas fontes de produtos naturais biologicamente ativos. 0 uso das plantas em práticas populares e tradicionais como medicamentos caseiros e comunitários, processo conhecido como medicina popular, nos remetem a seus princípios ativos como importantes substratos para o desenvolvimento de medicamentos ${ }^{1,2}$.

A procura por substâncias farmacologicamente ativas tem aumentado consideravelmente. Atualmente diversos grupos de pesquisa se dedicam ao estudo da atividade biológica de uma determinada classe de princípios ativos, utilizando a estratégia de modificação molecular para compreender seu mecanismo de ação, de maneira a permitir o desenvolvimento de medicamentos sintéticos mais seguros. Deve-se salientar que os estudos fitoquímicos de algumas plantas permitiram um grande avanço nas pesquisas de novos fármacos ao fornecer substâncias protótipo para o desenho de fármacos mais eficientes em determinados alvos moleculares ${ }^{3}$.

O isolamento dos constituintes ativos de plantas e a administração dos compostos puros é um modo pelo qual constituintes muito potentes podem ser transformados em produtos medicinais seguros de composição uniforme e consistente. Em outros casos, o objetivo pode ser melhorar a substância natural, aumentando suas propriedades desejáveis e minimizando os efeitos colaterais.

O estudo de plantas medicinais que possuam ação sobre o trato gastrintestinal assume grande importância uma vez que os medicamentos atualmente disponíveis para o tratamento destes distúrbios apresentam efeitos colaterais. $O$ uso de antiácidos pode causar constipação e diarréia ${ }^{4}$ e 0 uso crônico de fármacos anti-secretores tais como os antagonistas dos receptores $\mathrm{H}_{2}$ e os inibidores da bomba de prótons podem gerar problemas como gastrinemia ${ }^{5}$. Além disso, um estudo realizado por Arrais e colaboradores $(1997)^{6}$ demonstrou que a procura por medicamentos que tenham ação sobre o aparelho digestivo e o metabolismo é de $24 \%$, indicando que grande parte da população sofre de distúrbios gástricos, existindo grande necessidade de fármacos que apresentem menos efeitos colaterais e maior eficácia. A busca por princípios ativos que possam ser isolados ou servir de modelo para a síntese de novos fármacos é crescente e as pesquisas nesta área são promissoras.

Jatropha isabelli Müell Arg. pertence à família Euphorbiaceae, a qual constitui-se de 290 gêneros distribuídos principalmente em regiões tropicais e subtropicais, sendo muito bem representada na flora brasileira ${ }^{7,8}$. Em geral, o caule e as folhas contêm látex branco característico desta família 9 . O gênero Jatropha contém aproximadamente 170 espécies conhecidas $^{8}$ e compreende plantas de hábitos diferentes, como arbustos e árvores resistentes à seca ${ }^{10}$. As sementes das plantas contêm entre $40-60 \%$ de óleo ${ }^{11}$, o qual pode ser utilizado na fabricação de sabonetes e velas, onde substitui a parafina ${ }^{10,11}$.

Uma das espécies mais estudadas deste gênero é Jatropha curcas. Van den Berg e colaboradores $(1995)^{12}$ isolaram do látex de J. curcas um composto com atividade antitumoral (curcaciclina A). Outro composto isolado das sementes desta espécie (curcina) também

Saúde (Santa Maria), v.36, n.2, p. 19-28, jul./dez. 2010. Compostos isolados de jatropha isabelli (müell arg) como atividade gastroprotetora

20 ISSN 2236-5834 apresentou efeito antitumoral ${ }^{13}$. Rug e Rupel $(2000)^{14}$ verificaram a atividade molusquicida e larvicida dos diferentes extratos de J. curcas frente à Schistosoma mansoni e S. haematobium. Goonasekera e colaboradores $(1995)^{15}$ mostraram que os extratos de J. curcas possuem efeito abortivo. Mujumdar e Misar $(2004)^{16} \beta, 13 a \beta, 13 a$ verificaram a atividade antiinflamatória do extrato metanólico das raízes na prevenção do edema de pata induzido por carragenina. 
Villegas e colaboradores (1997) $)^{17}$ mostraram que a enzima proteolítica extraída do látex * comunicação pessoal apresentou atividade cicatrizante em feridas de ratos. O óleo de J. curcas possui ainda atividade lipásica, a qual pode catalizar reações de transesterificação para a preparação de biodiesel $11,18,19$.

Outra espécie com atividades biológicas importantes é a Jatropha elliptica. Calixto e colaboradores (1987) ${ }^{20}$ mostraram que o diterpeno jatrofona isolado desta espécie teve efeito inibitório direto nas preparações de músculo liso e cardíaco testadas e também que a potência pode variar de acordo com o estímulo ou tecido empregados para a indução da contração. Martini e colaboradores $(2000)^{21}$ avaliaram 0 composto jatrofona modulando a transmissão glutamatérgica como parâmetro de atividade antinociceptiva. Santos e colaboradores (1999 ${ }^{22}$ testaram a atividade molusquicida dos diterpenos isolados jatrofona e jatrofolona $A$ e $B$ frente a Biomphalaria glabrata. Lima e colaboradores $(2006)^{23}$ demonstraram a atividade antibacteriana contra Staphylococcus aureus. Além destes, outros estudos com os terpenos isolados desta espécie são reportados na literatura.

A espécie Jatropha isabelli é conhecida na medicina tradicional paraguaia como "yagua rova". O decocto e o infuso de seus rizomas são recomendados como digestivo, para tratar reumatismo, gota e induzir 0 aborto. A droga bruta é comercializada na forma de raízes secas $^{24}$. Em Santa Maria, RS, a planta é conhecida como "turubiti" ou "mamoneiro do campo", e seu uso é popularmente recomendado para dores nas costas. *

Alguns estudos científicos são reportados na literatura para a espécie J. isabelli como as atividades antiprotozoária e antiproliferativa. $\mathrm{Na}$ atividade antiprotozoária, SchmedaHirschmann e colaboradores (1996) ${ }^{25}$ testaram o diterpeno jatrofona isolado dos rizomas de $\mathrm{J}$. isabelli contra Leishmania spp e Tripanossoma cruzi in vitro, bem como contra Leishmania amazonensis in vivo. Na atividade antiprotozoária in vitro, a jatrofona foi completamente ativa contra os promastigotas de Leishmania spp e Tripanossoma cruzi. A atividade in vivo do diterpeno foi avaliada contra ratos infectados com Leishmania amazonensis. 0 composto jatrofona reduziu significativamente o diâmetro das lesões em comparação com os ratos controle, que foram tratados com antimoniato de meglumina. Entretanto, metade dos ratos que receberam jatrofona $(25 \mathrm{mg} / \mathrm{Kg} / \mathrm{dia})$ morreram durante o experimento. Assim, a toxicidade do diterpeno jatrofona torna seu uso impróprio para a terapia da leishmaniose. Theodulóz e colaboradores $(2009)^{26}$ avaliaram a atividade antiproliferativa dos diterpenos jatrofolona A e B e 16 derivados semi-sintéticos dos mesmos, do composto jatrofona e três derivados deste em culturas de células humanas. As células utilizadas foram fibroblastos de pulmão normal (MRC5), adenocarcinoma gástrico (AGS), leucemia (HL-60), câncer de pulmão (SK-MES-1) e carcinoma da bexiga (J82). 0 composto jatrofolona A foi inativo contra todas as linhagens de células tumorais, mas sua acetilação originou um composto com atividade antiproliferativa. 0 composto jatrofolona B foi ativo contra todas as linhagens de células tumorais, e seus derivados apresentaram efeitos distintos sobre as linhagens de células selecionadas. 0 composto jatrofona mostrou forte atividade anticancerígena enquanto que seus derivados $9 ß$, 13a-diidroxiisabeliona e 13a-hidroxi-acetoxiisabelliona-9ß foram menos ativos.

Em meio a estes estudos, os trabalhos realizados sobre a atividade gastroprotetora da planta merecem destaque. A espécie teve os constituintes terpênicos isolados e modificados estruturalmente com a finalidade de verificar a atividade gastroprotetora destes compostos e seus derivados. Este trabalho tem como objetivo reunir estes estudos visando avaliar 0 potencial gastroprotetor desta planta.

Saúde (Santa Maria), v.36, n.2, p.19-28, jul./dez. 2010

Fröhlich, J. K.; Boligon, A. A.; Feltrin, A. C.; Janovik, V.; Froeder, A. L. F.; Athayde, M. L. ISSN 2236-5843 


\section{Compostos isolados de Jatropha isabelli Müell Arg.}

Os constituintes isolados dos rizomas de $\mathrm{J}$. isabelli consistem em terpenóides. Pertino e colaboradores (2007) ${ }^{24}$ isolaram o ácido acetil aleuritólico (1), o ácido ciperenóico (2) e 0 diterpeno jatrofona (3), previamente isolado por Schmeda-Hirschmann e colaboradores em $1996^{25}$ quando este composto foi identificado por métodos espectroscópicos e difração de raios X. Os compostos jatrofolona A (4) e B (5), um novo derivado jatrofona (6) denominado 9ß, 13diidroxiisabelliona, seu derivado acetilado (6a) e um monoterpeno 1,4-epoxi-p-mentan-2-ol (7) também foram isolados por Pertino e colaboradores (2007) ${ }^{24}$. Estes compostos foram caracterizados por espectrometria de massa e suas estruturas podem ser vistas na Figura 1.

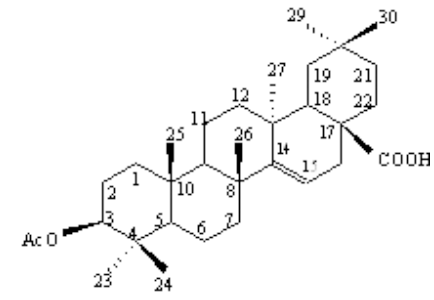

Ácido acetil aleuritólico (1)

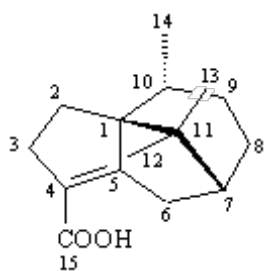

Ácido ciperenóico (2)

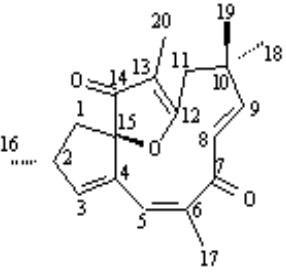

Jatrofona (3)

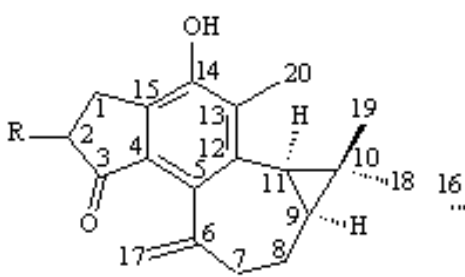

R: ß CH3; $a \mathrm{H}$ Jatrofolona A (4) R: a CH3; ß H Jatrofolona B (5)

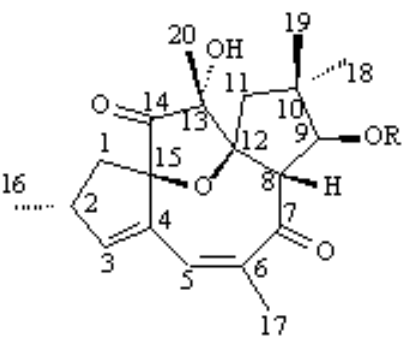

R: $\mathrm{H}$ Novo derivado Jatrofona (6) R: Ac Jatrofona Acetilada (6a)<smiles>CC(C)C12CCC(CC1)C(O)C2</smiles>

1,4-epóxi- p-mentan -2-ol (7)

Figura 1 - Estrutura dos terpenos isolados de Jatropha isabelli Müell Arg. Fonte: Pertino et al, 24.

\section{Atividades gastroprotetoras descritas para Jatropha isabelli Müell Arg.}

O efeito gastroprotetor dos terpenos ácido acetil aleuritólico (1), do diterpeno jatrofona (3), das jatrofolonas A e B (4 e 5), do composto 9ß, 13 diidroxiisabelliona (6) e seu derivado acetilado (6a) foi testado por Pertino et al. 24. Este efeito foi avaliado seguindo o modelo de indução de lesão gástrica com ácido clorídrico/etanol em ratos, tendo como padrão 0 lansoprazol. Os experimentos dose-resposta foram avaliados com doses orais de 25, $50 \mathrm{e}$ $100 \mathrm{mg} / \mathrm{Kg}$ para os compostos 1, 3 e 5 e os resultados expressos em porcentagem em relação ao controle. 0 triterpeno ácido acetil aleuritólico (1) apresentou efeito gastroprotetor já na menor dose, reduzindo as lesões em cerca de $50 \%$ quando comparado ao lansoprazol. 0 diterpeno jatrofona (3) apresentou um forte efeito gastroprotetor com diferenças não significantes entre as doses de 25,50 ou $100 \mathrm{mg} / \mathrm{Kg}$ e com redução das lesões gástricas de 88

Saúde (Santa Maria), v.36, n.2, p. 19-28, jul./dez. 2010.

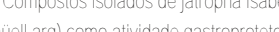
22 | a $93 \%$. As jatrofolonas A e B (4 e 5) que diferem somente na estereoquímica do grupamento metila do C-16, mostraram notáveis diferenças na avaliação gastroprotera. Enquanto que a jatrofolona A apresentou uma dose-resposta proporcional, com efeito máximo ( $54 \%$ de redução de lesão) na maior dose $(100 \mathrm{mg} / \mathrm{Kg})$, a jatrofolona B mostrou uma ação forte em todas as 
doses, reduzindo as lesões de 83-91\%. O diterpeno 9ß, 13 dihidroxiisabelliona (6) e seu derivado acetilado (6a) foram avaliados para dose única de $25 \mathrm{mg} / \mathrm{Kg}$. $O$ efeito gastroprotetor do primeiro foi baixo, apenas $35 \%$, mas a presença do acetato melhora sua atividade, reduzindo as lesões em $65 \%$. Os efeitos dos compostos jatrofona (3) e jatrofolona B (5) na dose de $25 \mathrm{mg} / \mathrm{Kg}$ (88 e $83 \%$ respectivamente) foram mais elevados que do composto referência lansoprazol na dose de $20 \mathrm{mg} / \mathrm{Kg}(73 \%)$.

A citotoxicidade dos compostos isolados foi avaliada através da viabilidade celular de fibroblastos derivados do pulmão humano (MRC-55) e células AGS (células do epitélio gástrico humano). Os resultados foram expressos em $I_{50}$ (concentração que produz um efeito inibitório de $50 \%$ da viabilidade celular). 0 composto jatrofona (3) foi altamente citotóxico para ambas linhagens celulares obtendo $\mathrm{IC}_{50}$ de $2,5 \mu \mathrm{M}$ para células AGS e de $2,8 \mu \mathrm{M}$ para fibroblastos. Jatrofolona $B(5)$ e ácido acetil aleuritólico (1) não foram citotóxicos $\left(I_{50}>1000 \mu \mathrm{M}\right)$, enquanto

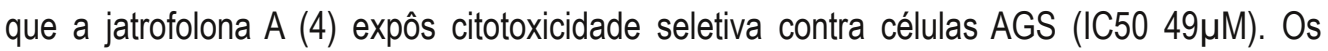
efeitos do novo derivado jatrofona (6) e seu acetato (6a) apresentaram-se similares, com maior

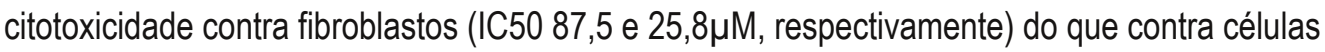
AGS $\left(I_{50} 200 \text { e } 92,8 \mu \mathrm{M} \text {, respectivamente }\right)^{24}$.

Ainda levando em consideração os terpenos isolados dos rizomas de J. isabelli, Pertino et al. ${ }^{27}$, estudaram a atividade gastroprotetora e 0 efeito citotóxico do sesquiterpeno ácido ciperenóico e seus derivados semi-sintéticos (Figura 2). 0 efeito gastroprotetor do ácido ciperenóico e dos sete derivados foi avaliado seguindo o modelo de indução de lesão gástrica com ácido clorídrico/etanol em ratos tendo como padrão o lansoprazol. Os resultados foram expressos em porcentagem em relação ao controle. Doses de 50 e 100mg/Kg de ácido ciperenóico mostraram um efeito gastroprotetor dose-dependente, com redução das lesões de 45 e 75\%, respectivamente. Os derivados sesquiterpênicos do ácido ciperenóico (compostos de 1-8) foram preparados e suas atividades gastroprotetoras foram testadas na concentração de $50 \mathrm{mg} / \mathrm{Kg}$. Nesta concentração, o derivado 8 (formado pela mistura dos isômeros $8 \mathrm{a}$ e 8b) apresentou o melhor efeito gastroprotetor, reduzindo as lesões gástricas em $86 \%$, tendo assim, um efeito maior que o controle lansoprazol $(20 \mathrm{mg} / \mathrm{Kg})$. O efeito gastroprotetor dos derivados 1 , 4, 5 e 6, teve o mesmo alcance, reduzindo as lesões gástricas de $72-77 \%$. A citotoxicidade dos compostos foi avaliada através da viabilidade celular de fibroblastos derivados do pulmão

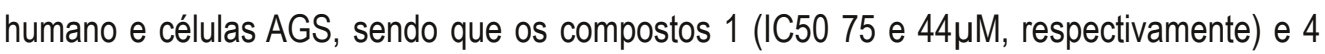
$\left(\mathrm{IC}_{50} 75\right.$ e $\left.48 \mu \mathrm{M}\right)$ tiveram os maiores efeitos citotóxicos. 0 melhor efeito gastroprotetor associado a uma baixa citotoxicidade foi encontrado nos derivados 8,3 e 7 . 


\begin{tabular}{|c|c|}
\hline Compostos & $\mathrm{R}$ \\
\hline 1 & $\mathrm{CH}_{2} \mathrm{OH}$ \\
\hline 2 & $\mathrm{CH}_{2} \mathrm{OCOCH}_{3}$ \\
\hline 3 & $\mathrm{COOH}$ \\
\hline 4 & $\mathrm{COOCH}_{3}$ \\
\hline 5 & $\mathrm{CONHCH}_{2} \mathrm{CH}_{3}$ \\
\hline 6 & $\mathrm{CONHCH}_{2} \mathrm{CH}_{2} \mathrm{CH}_{2} \mathrm{CH}_{3}$ \\
\hline 7 & CONHPhOCH3 \\
\hline
\end{tabular}

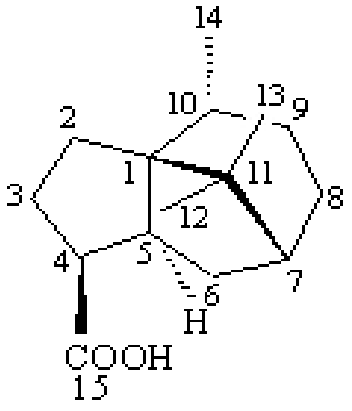

Isômero 8a

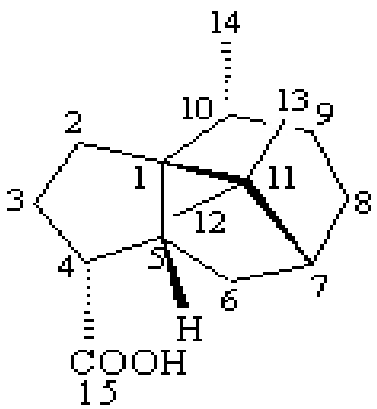

Isômero 8b

Figura 2 - Estrutura dos compostos 1-8 derivados do ácido ciperenóico. Fonte: Pertino et al, 27.

Pertino et al.28 também avaliaram o efeito gastroprotetor e citotóxico dos diterpenos jatrofolona $A$ e $B$ de seus derivados semi-sintéticos (Figura 3). $O$ efeito gastroprotetor foi avaliado seguindo o modelo de indução de lesão gástrica com ácido clorídrico/etanol em ratos, tendo como padrão o lansoprazol. A citotoxicidade foi avaliada através da viabilidade celular de fibroblastos derivados do pulmão humano e células AGS.

O composto 1 (jatrofolona A) foi menos ativo que o composto 8 (jatrofolona B) na prevenção do aparecimento de úlceras gástricas. Na dose de $25 \mathrm{mg} / \mathrm{Kg} 0$ composto 8 reduziu as lesões em $83 \%$ enquanto que o composto 1 reduziu em apenas $36 \%$. Na dosagem de $100 \mathrm{mg} / \mathrm{Kg}$ todos os derivados do composto 1 (compostos 2-7) foram ativos. Os compostos 4-6 mostraram uma atividade similar a jatrofolona $A$, enquanto que os derivados 2, 3 e 7 tiveram maior atividade. Considerando os derivados do composto 8 (compostos 9-14) e os compostos derivados da mistura das jatrofolonas A e B (compostos 15-18), na concentração de $25 \mathrm{mg} / \mathrm{Kg}$, (müell arg) como atividade gastroprotetora os derivados 10, 17 e 18 mostraram o melhor efeito gastroprotetor, enquanto os compostos 14 e 15 foram os menos ativos e o composto 16 foi isento de atividade. Com referência a citotoxicidade, a jatrofolona $B$ não foi tóxica contra células $A G S$ e fibroblastos $\left(I C_{50}>1000 \mu \mathrm{M}\right)$, enquanto que a jatrofolona $A$ foi citotóxica apenas contra células AGS $\left(I_{50} 49 \mu \mathrm{M}\right)$. 

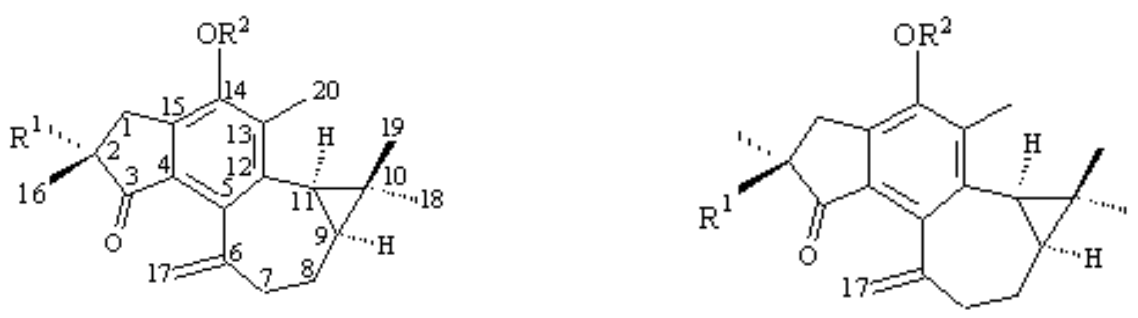

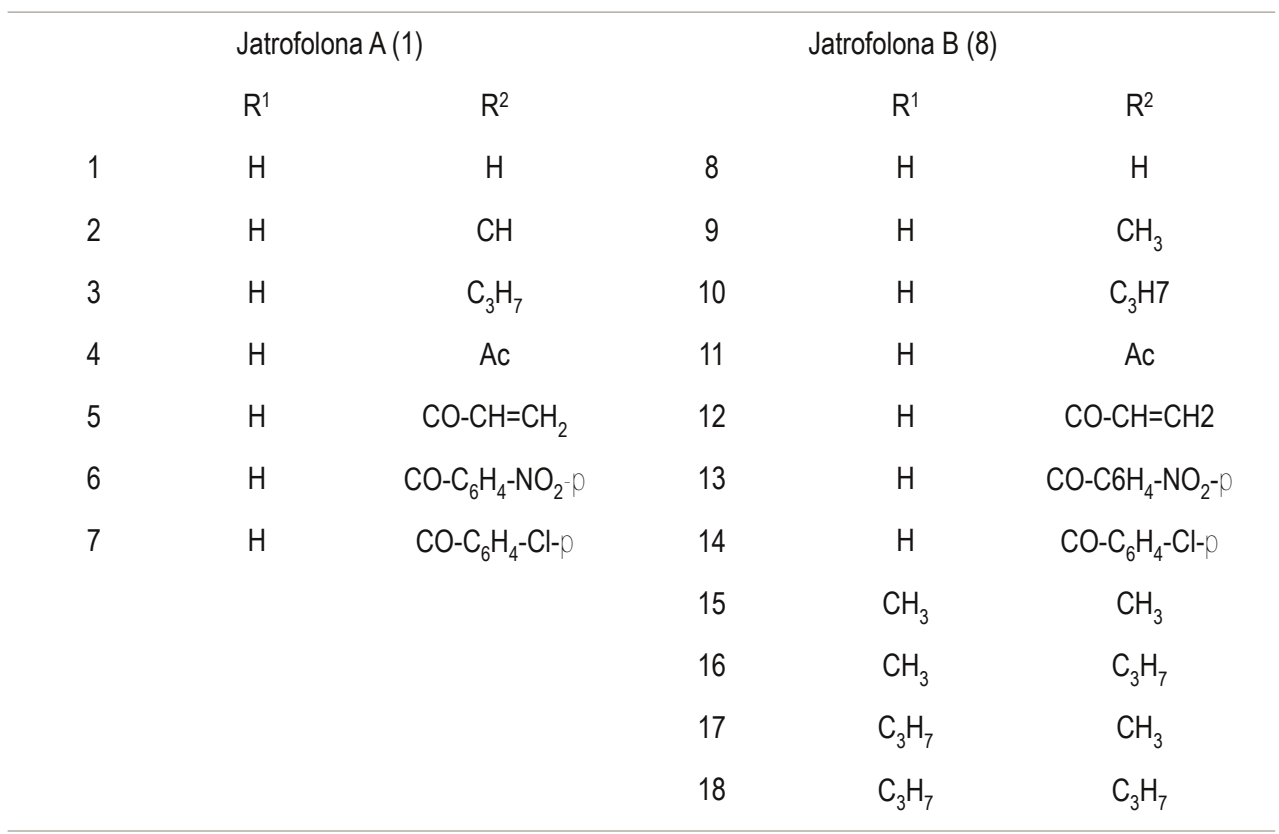

Figura 3 - Estruturas da jatrofolona A (1), jatrofolona B (8) e seus derivados semi-sintéticos.

Fonte: Pertino et al, 28.

\section{Considerações Finais}

Muitos de nossos medicamentos atuais são derivados de fontes naturais. Observa-se que aproximadamente 119 substâncias químicas extraídas de cerca de 90 espécies de plantas superiores são usadas na medicina e no mundo, e $77 \%$ dessas são derivadas de plantas usadas na medicina tradicional.

Os ensaios biológicos descritos na literatura para alguns compostos ativos isolados a partir das raízes de J. isabelli revelam um potente efeito gastroprotetor não relatado pela medicina popular, o que permite uma ampliação dos conhecimentos benéficos desta espécie.

A jatrofolona $B$ merece destaque, pois não apresentou toxicidade frente a fibroblastos e células AGS e reduziu as lesões ulcerosas em $83 \%$ já na menor dose testada, se comparado com o padrão lansoprazol. Estes dados indicam que a jatrofolona $B$ é um terpenóide de efeito gastroprotetor considerável, merecendo estudos mais aprofundados a seu respeito com 0 objetivo de sintetizá-lo quimicamente para o desenvolvimento de um novo fármaco. Para 0 desenvolvimento de um medicamento protetor da mucosa estomacal a partir deste ativo, ainda são necessários um maior número de pesquisas a nível farmacológico, toxicológico e molecular para permitir a constatação do seu mecanismo de ação total ou parcial. A avaliação toxicológica deve comprovar a segurança do ativo e os estudos de farmacologia pré-clínica e farmacologia clínica, devem ser realizados segundo as normas que regem os processos de validação de fármacos puros ${ }^{29}$.

Saúde (Santa Maria), v.36, n.2, p.19-28, jul./dez. 2010

Fröhlich, J. K.; Boligon, A. A.; Feltrin, A. C.; Janovik, V.; Froeder, A. L. F.; Athayde, M. L. ISSN $2236-5843$ 
Os estudos já comprovados para J. isabelli demonstram apenas uma parte do potencial desta planta para o desenvolvimento de medicamentos gastroprotetores. Entretanto, persiste ainda a necessidade de demonstrar, através de pesquisas, outras atividades farmacológicas, as quais remetem o seu uso popular para tratar gota e reumatismo.

J. isabelli é uma planta bastante promissora do ponto de vista químico e farmacológico, podendo demonstrar outras propriedades interessantes a partir do maior número de pesquisas a seu respeito, uma vez que pouco se sabe da atividade química e biológica desta planta.

\section{Referências Bibliográficas}

1. Maciel MAM, Pinto AC, Veiga JR VF, Grynberg NF, Echevarria A. Plantas Medicinais: a necessidade de estudos multidisciplinares. Química Nova 2002; 25 (3): 429-438.

2. Calixto JB. Fitofármacos no Brasil: agora ou nunca! Ciência hoje 1997; 21 (1234): 26-30.

3. Barreiro EJ. Produtos Naturais Bioativos de Origem Vegetal e o Desenvolvimento de Fármacos. Química Nova 1990; 13(1): 29-39.

4. Rang HP, Dale MM, Ritter JM. Farmacologia. $4^{a}$ ed. Rio de Janeiro: Guanabara Koogan, 2001.

5. Garnett, WR. Considerations for long-term use of proton-pump inhibitors. American Journal of Health-System Pharmacy. 1998; 55(21):2268-2279.

6. Arrais PSD, Coelho HLL, Batista MCDS, Carvalho ML, Righi RE, Arnau JM. Perfil da automedicação no Brasil. Revista de Saúde Pública. 1997; 31: 71-77.

7. Weberling F, Schwantes HO. Taxonomia Vegetal. $4^{a}$. ed. São Paulo: Editora Pedagógica e Universitária Ltda, 1986.

8. Joly AB. Botânica: introdução à taxonomia vegetal. 12ª . ed. São Paulo: Companhia Editora Nacional, 1998.

9. Schultz A. Introdução à botânica sistemática. 5ª.ed. Porto Alegre: Editora da Universidade, v.2, 1985.

10. Kumar A, Sharma S. An evaluation of multipurpose oil seed crop for industrial uses (Jatropha curcas L.): A review. Industrial Crops and Products 2008; 28(1): 1-10.

11. Shah S, Sharma S, Gupta MN. Biodiesel preparation by lipase-catalysed transesterification of Jatropha oil. Energy e Fuels 2004; 18(1): 154-159.

12. Van den Berg AJ, Horsten SF, Kettenes Van den Bosch JJ, Kroes BH, Beukelman CJ, Loeflang BR, Labadie RP. Curcacycline A: a novel cyclic octapeptide isolated from the latex of Jatropha curcas Linn. FEBS Lettes.1995; 358:215-218.

13. Lin J, Yan F, Tang L, Chen F. Antitumor effects of curcin from seeds of Jatropha curcas. Acta Pharmacology. 2003; 24 (3): 241-246.

14. Rug M, Ruppel A. Toxic activities of the plant Jatropha curcas against intermediate snail hosts and larvae of schistosomes. Tropical Medicine and International Health. 2000; 5(6): 423-430.

15. Goonasekera MM, Gunawardana VK, Jayasena K, Mohammed SG, Balasubramaniam S. Pregnancy terminating effect of Jatropha curcas in rats. Journal of Ethnopharmacology. 1995; 47: 117-123.

16. Mujumdar AM, Misar AV. Anti-inflamatory activity of Jatropha curcas roots in mice and rats. Journal of Ethnopharmacology. 2004; 90: 11-15.

Compostos isolados de jatropha isabell (müell arg) como atividade gastroprotetora

17. Villegas LF, Fernandez ID, Maldonado H, Torres R, Zavaleta A, Vaisberg AJ, Hammond GB. Evaluation of the wound-healing activity of selected traditional medicinal plants from Peru. Journal of Ethnopharmacology. 1997; 55: 193-200. 
18. Staubman R, Ncube I, Gübitz GM, Steiner W, Read JS. Esterase and lipase activity in Jatropha curcas L. Seeds. Journal of Biotechnology. 1999; 75(2-3): 117-126.

19. Openshaw k. A review of Jatropha curcas: an oil plant of unfulfilled promise. Biomass and Bioenergy. 2000; 19(1):1-15.

20. Calixto JB, Sant'Ana AEG. Pharmacological analysis of the inhibitory effect of jatrophone, a diterpene isolated from Jatropha elliptica, on smooth and cardiac muscles. Phytotherapy Research. 1987; 1(3): 122-126.

21. Martini LH, Souza CR, Marques PB, Calixto JB, Yunes RA, Souza DO. Compounds Extracted from Phyllantus and Jatropha elliptica Inhibit the Binding of [3H]Glutamate and [3H]GMP-PNP in Rat Cerebral Cortex Membrane. Neurochemical Research. 2000; 25(2): 211-215.

22. Santos AF, Sant'Ana AEG. Molluscicidal activity of the diterpenoids jatrophone and jatropholones A and B isolated from Jatropha elliptica (Pohl). Phytotherapy Research. 1999; 13(8): 660-664.

23. Lima MRF, Luna JS, Santos AF, Andrade MCC, Sant'Ana AEG, Genet JP, Marquez B, Neuville L, Moreau N. Anti-bacterial activity of some Brazilian medicinal plants. Journal of Ethnopharmacology. 2006; 105 (1-2): 137147.

24. Pertino M, Schmeda-Hirschmann G, Rodriguez JA, Theoduloz C. Gastroprotective effect and citotoxicity of terpenes from Paraguayan crude drug "yagua rova" (Jatropha isabelli). Journal of Ethnopharmacology 2007a; 111(3): 553-559.

25. Schmeda-Hirschmann G, Razmilic I. Antiprotozoal activity of Jatrogrossidione from Jatropha grossidentata an Jatrophone from Jatropha isabelli. Phytotherapy Research 1996; 10: 375-378.

26. Theoduloz C, Rodriguez JA, Pertino M, Schmeda-Hirschmann G. Antiproliferative activity of the diterpenes jatrophone and jatropholone and their derivatives. Planta Medica. 2009; 75(14): 1520-1522.

27. Pertino M, Schmeda-Hirschmann G, Rodriguez JA, Theoduloz C, Razmilic I. Gastroprotective activity and citotoxic effect of cyperenoic acid derivatives. Journal of Pharmacy and Pharmacology 2006; 58: 1507-1513.

28. Pertino M, Schmeda-Hirschmann G, Rodriguez JA, Theoduloz C. Gastroprotective effect and citotoxicity of semisynthetic Jatropholone derivatives. Planta Med 2007b; 73: 1095-1100.

29. Yunes RA, Pedrosa RC, Filho VC. Fármacos e Fitoterápicos: a necessidade do desenvolvimento da indústria de fitoterápicos e fitofármacos no Brasil. Química Nova 2001; 24(1): 147-152.

\section{Endereço para correspondência}

Janaina Kieling Fröhlich - Universidade Federal de Santa Maria, Prédio 26, sala 1411, Bairro Camobi, Santa Maria.Tel: (055) 3220-9618

E-mail: janafrohlich@yahoo.com.br

Recebido em 30 de novembro de 2010.

Aceito em 07 de abril de 2011. 